\title{
Dienel, Hans-Liudger, Schiedt, Hans-Ulrich, Die moderne Strasse : Planung, Bau und Verkehr vom 18. bis zum 20. Jahrhundert
}

Denis Bocquet

\section{OpenEdition}

\section{Journals}

Édition électronique

URL : http://journals.openedition.org/ifha/6562

DOI : $10.4000 /$ ifha. 6562

ISSN : 2198-8943

\section{Éditeur}

IFRA - Institut franco-allemand (sciences historiques et sociales)

Référence électronique

Denis Bocquet, « Dienel, Hans-Liudger, Schiedt, Hans-Ulrich, Die moderne Strasse : Planung, Bau und Verkehr vom 18. bis zum 20. Jahrhundert», Revue de l'IFHA [En ligne], Date de recension, mis en ligne le 01 janvier 2011, consulté le 22 septembre 2020. URL : http://journals.openedition.org/ifha/6562 ; DOI : https://doi.org/10.4000/ifha.6562

Ce document a été généré automatiquement le 22 septembre 2020.

(C)IFHA 


\title{
Dienel, Hans-Liudger, Schiedt, Hans- Ulrich, Die moderne Strasse : Planung, Bau und Verkehr vom 18. bis zum 20. Jahrhundert
}

\author{
Denis Bocquet
}

Les études de Bernard Lepetit ont montré combien l'attention à la modernisation des réseaux viaires pouvait constituer une entrée déterminante dans la compréhension des processus de construction des appareils étatiques entre époque moderne et contemporaine et dans l'évaluation du changement majeur dans le rapport au territoire qui s'est joué entre ces périodes. Les coordinateurs de ce livre collectif, qui présentent leur projet dans une introduction problématisée, tout en ne se réclamant pas de l'héritage intellectuel de B. Lepetit, proposent une perspective transnationale sur ce sujet. $\mathrm{N}$. Longen s'intéresse ainsi à la transformation des travaux d'origine féodale de type corvée entre les XVIIIe et XIXe siècles et au rôle de cet héritage dans le financement des travaux d'infrastructure dans l'électorat de Trèves. U. Müller étudie quant à elle la politique routière prussienne au XIXe siècle, apportant notamment d'intéressants éléments sur les processus de professionnalisation et de spécialisation de l'administration, avec une réflexion sur le rapport entre État central et communautés locales. La perspective adoptée par G. Mom est davantage transnationale : il s'agit, pour cette fois le XXe siècle, de comparer la manière avec laquelle les différents États européens ont accompagné le mouvement massif de motorisation des sociétés. Son étude se concentre sur l'évolution des appareils centraux de planification routière. L'approche de J. Ludwig ouvre en revanche, avec une attention à l'industrie du basalte en Allemagne, sur la dimension quasi-minière du développement des réseaux routiers. Cela amène le chercheur à une histoire des grandes entreprises de travaux publics, comme Strabag. Le chapitre de R. Ruppmann, consacré à la région de Francfort, « Stadt der Strassen ", ramène le livre vers une dimension plus urbaine et de réflexion métropolitaine. Il s'agit d'analyser la place du réseau autoroutier dans l'évolution de l'idée même d'ensemble urbain pour cette région. M. Hascher, pour le XXe siècle, étudie 
pour sa part l'évolution des méthodes et conception de péage pour le financement des infrastructures routières. Il part des idées de Robert Bosch en 1908 pour conclure avec l'étude des portiques introduits sur les autoroutes allemandes dans les années 2000. B. Kreuzer consacre un chapitre à l'histoire des autoroutes autrichiennes, avec une perspective comparatiste par rapport aux États-Unis, à l'Allemagne, à l'Italie, à la France et au Bénelux. J. Olivia présente la modernisation du réseau routier tchécoslovaque dans l'entre-deux guerres, et $\mathrm{M}$. Wagner s'attache à étudier l'avancée du réseau mexicain dans cette même période. Le chapitre d'A. Gall revient à l'échelle des bureaux d'étude, et s'intéresse à l'évolution en Allemagne des conceptions de la planification, entre État fédéral et Länder pour les années 1967-1974. T. Zeller adopte quant à lui une perspective d'histoire paysagère pour analyser le rôle de la route dans la construction en Allemagne et aux États-Unis d'un nouvel imaginaire de la montagne. La fin du livre est consacrée à la logistique et au secteur du fret, avec des études de $\mathrm{R}$. Vahrenkamp sur le développement du transport routier au début du XXe siècle, de M. Hesse sur le thème ville et logistique, et de $\mathrm{P}$. Erker sur les entreprises allemandes de fret routier. Si au total on peut regretter qu'il manque à ce livre une dimension plus grande de réflexion historiographique concertée, il demeure qu'il recèle d'un certain nombre d'études extrêmement bien documentées qui sauront fournir aux tenants de telles discussions des cas d'études précieux.

Denis Bocquet (École des Ponts / ParisTech, LATTS) 